\title{
Deformation and stress analysis of catenary shell of revolution
}

\author{
Bohua Sun ${ }^{a, 1}$ \\ ${ }^{a}$ School of Civil Engineering \& Institute of Mechanics and Technology, Xi'an University of Architecture and Technology, Xi'an 710055, China
}

\section{ARTICLE INFO}

\section{Keywords:}

Catenary, surface of revolution, Gauss curvature, minimal surface, shells, deformation, stress, Maple

\begin{abstract}
A B S T R ACT
The catenary shells of revolution are widely used in constructions due to their unique mechanics' feature. However, no publications on this type of shells can be found in the literature. To have a better understanding of the deformation and stress of the catenary shells of revolution, we formulate the principal radii for two kinds of catenary shells of revolution and their displacement type governing equations. Numerical simulations are carried out based on both Reissner-Meissner mixed formulations and displacement formulations. Our investigations show that both deformation and stress response of elastic catenary shells of revolution are sensitive to its geometric parameter $c$, and reveal that the mechanics of the catenary shells of revolution does much better than the spherical shells. Two complete codes in Maple are provided.
\end{abstract}

\section{Contents}

1 Introduction

2 Bending theory formulations of the shells of revolution

2.1 Reissner-Meissner mixed formulation of the shells of revolution . . . . . . . . . .

2.2 Displacement formulation of the shells of rev-

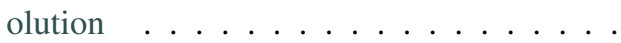

2.3 The sign convention of different formulations and physics units ........

3 The principal radii of curvature of the catenary shells of revolution

3.1 The principal radii of curvature of the catenary shells of revolution $1 . . . . .$. . .

3.2 The principal radii of curvature of the catenary shells of revolution $2 \ldots . . .$.

4 Numerical studies for the deformation and stress of a truncated catenary shell of revolution 1

4.1 Numerical results based on the displacement formulations ............ 6

4.2 Numerical results based on the Reissner-Meissner formulations .............. 6

4.3 Results validation . . . . . . . . . 6

5 Numerical studies for the deformation and stress of a catenary shell of revolution 2 Sun)

\footnotetext{
${ }^{*}$ Corresponding author.

sunbohua@xauat.edu.cn (.B. Sun); Mobile:+86 15001102877 (.B
}

- imt.xauat. edu.cn (.B. Sun)
6 The catenary shell of revolution vs. the spherical shells

7 Analytical investigation on the catenary shells of revolution 2

7.1 Solutions for the Poisson ratio $v=0$. . . 9

7.2 Approximation in the vicinity of the apex . 10

8 Conclusions

\section{Introduction}

A surface of revolution is a surface generated by rotating a 2-D curve about an axis. The resulting surface therefore always has azimuthal symmetry about the axis. One kind of the surface of revolution has a minimal surface area, which are sometimes also called catenoid. A popular example of the catenoid is a soap film "stretched" over two wire discs as shown in Fig.1.

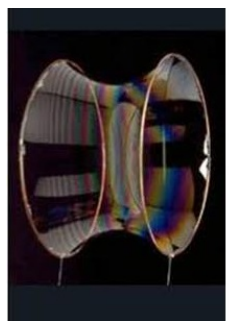

Figure 1: The soap bubble "stretched" over two wire discs.

The catenary is the shape that makes the potential energy of a suspended uniform flexible chain minimum. If $m$ denotes the mass as per unit length of the chain and $g$ the 
gravitational constant, the potential energy of the chain between the poles is $J[z(y)]=\int_{a}^{b} m g z(y) \sqrt{1+z^{\prime 2}(y)} d y$, its variational condition $\delta \boldsymbol{J}=0$, leads to the solution of the catenary $z=c \cosh \frac{y}{c}$, where $c$ is a positive constant. The catenary shape of the chain is shown in Fig.2.

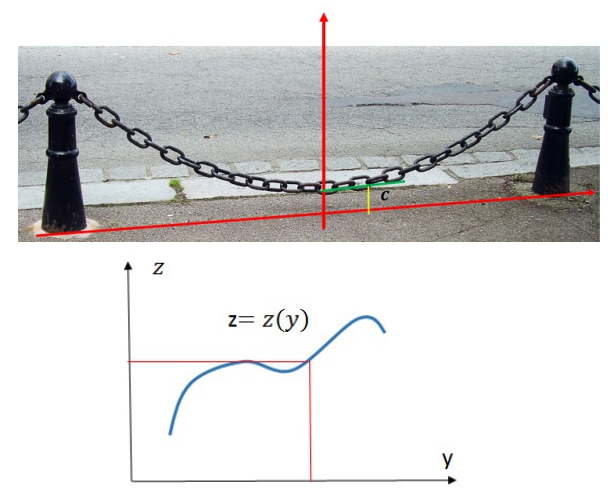

Figure 2: The uniform chain takes the catenary shape under the gravitation: $z=c \cosh y / c$.

Two kind of surfaces can be generated by rotating the catenary curve about axis $y$ and $z$, respectively, as shown in Fig.3, where the surface 2 is a minimal surface of revolution while the surface 1 is not. In this paper we will study the shells of revolution that use the two surfaces as their middle surfaces. It is expected that the mechanics of those two shells of revolution are quite different due to their different geometry.

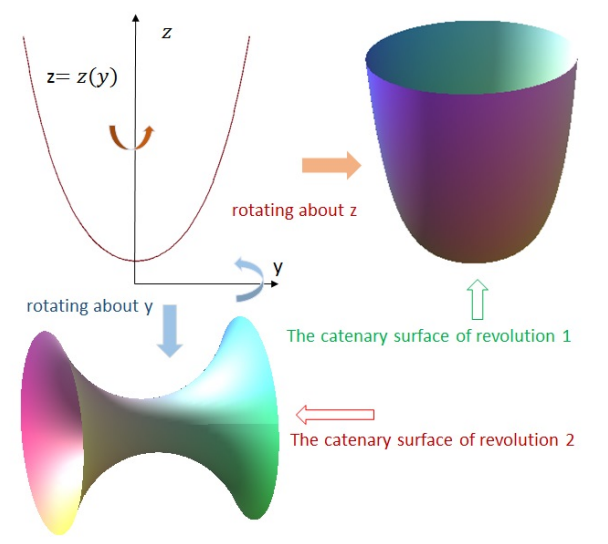

Figure 3: Two kind of surfaces are generated by rotating the catenary curve about axis $y$ and $z$. The catenary surface of revolution 2 is a minimal surface while the surface 1 is not.

Although the shell structures used the surface of revolution as their middle surface are widely used in constructions, the authors could not find any scientific publications that applied the theory of shells to the analysis of the catenary shells of revolution. To fill the vacancy of the research on the cate- nary shells of revolution, we study the small deformation of the shell 1 and 2 shown in Fig. 3 by using the bending theory of shells (Timoshenko and Woinowsky-Krieger, 1959; Novozhilov, 1959; Gol'denveizer, 1961; Kraus, 1967; Flügge, 1973; Calladine, 1983; Reddy, 2007; Audoly and Pomeau, 2010; Sun, 2012; Zingoni, 2017; Ugural, 2018; Sun, 2021).

The content is organised as follows: Section 2 presents Reissner-Meissner mixed formulation and displacement formulations of the bending theory of shells of revolution. Section 3 formulates and proposes the principal radii of the catenary surface of revolution. Section 4 and 5 carry out some numerical investigations on truncated catenary shells of revolution and results validation. Section 6 presents a comparison between the spherical shells and the catenary shells of revolution. Section 7 derives Reissner-Meissner governing equations for the catenary shells of revolution 2 and propose exact solutions when the Poisson ratio is neglected. Section 8 concludes with perspectives. Last but not least, the appendix provides two complete codes in Maple.

\section{Bending theory formulations of the shells of revolution}

When the linear problem was first studied, high-order and complicated governing equations of a torus under symmetric loads were reduced to lower-order, ordinary differential equations (ODE) by Hans Reissner (Reissner, 1912) when he was a professor at ETH in Switzerland. His colleague at ETH, Meissner (Meissner, 1915) derived a complexform equations for the shell of revolution. Hence, the first complex-form equation of the shells of revolution including tori is called the Reissner-Meissner equation, which is an ODE system for the shear force $Q$ and the rotation $\chi$ (Flügge, 1973). In 1959, Novozhilov (Novozhilov, 1959) published his celebrated monograph on the complex-form theory of shells.

\subsection{Reissner-Meissner mixed formulation of the shells of revolution}

In the theory of shells of revolution under axisymmetric load, according to Timoshenko and Woinowsky-Krieger 1959 (Timoshenko and Woinowsky-Krieger, 1959), the decisive step was the introduction of $Q_{\phi}$ and $\chi$ as unknowns by H. Reissner 1912 (Reissner, 1912). The idea has been much extended by E. Meissner 1925 (Meissner, 1915). The formulation of Reissner-Meissner of shells of revaluation with constant thickness can be found in the masterpieces of Flügge 1973 (Flügge, 1973).

For convenience of readers, all quantities notation and definition in this section please refers to well-known book of Flügge (Flügge, 1973).

The balance equations are:

$$
\left[\frac{r_{2}}{r_{1}} \cot \phi+\frac{d}{d \phi}\left(\frac{r_{2}}{r_{1}}\right)\right] \frac{d \chi}{d \phi}-\left(\frac{r_{1}}{r_{2}} \cot ^{2} \phi+v\right) \chi
$$




$$
\begin{aligned}
& +\frac{r_{2}}{r_{1}} \frac{d^{2} \chi}{d \phi^{2}}=\frac{1}{B} r_{1} r_{2} Q_{\phi}, \\
& \frac{r_{2}}{r_{1}} \frac{d^{2}}{d \phi^{2}}\left(r_{2} Q_{\phi}\right)-\left(\frac{r_{1}}{r_{2}} \cot ^{2} \phi-v\right)\left(r_{2} Q_{\phi}\right) \\
& +\left[\frac{r_{2}}{r_{1}} \cot \phi+\frac{d}{d \phi}\left(\frac{r_{2}}{r_{1}}\right)\right] \frac{d}{d \phi}\left(r_{2} Q_{\phi}\right) \\
& =-B\left(1-v^{2}\right) r_{1} \chi+\operatorname{Pg}(\phi),
\end{aligned}
$$

where $\chi$ is the angle by which an element $r d \phi$ of the meridian rotates during deformation; $r=r_{2} \sin \phi$, the load term $P$ is constant to be determined by the value of $N_{\phi \phi}(\pi / 2)=$ $-\frac{P}{2 \pi r_{2}}$ and

$$
g(\phi)=\frac{1}{2 \pi \sin ^{2} \phi}\left[\frac{r_{1}^{2}-r_{2}^{2}}{r_{1} r_{2}} \cot \phi+\frac{d}{d \phi}\left(\frac{r_{2}}{r_{1}}\right)\right] .
$$

The resultant membranae forces $N_{\phi \phi}$ can be represented by shear force $Q_{\phi}$ :

$$
\begin{aligned}
N_{\phi \phi} & =-Q_{\phi} \cot \phi-\frac{P}{2 \pi r_{2}} \frac{1}{\sin ^{2} \phi}, \\
N_{\theta \theta} & =-\frac{1}{r_{1}} \frac{d}{d \phi}\left(r_{2} Q_{\phi}\right)+\frac{P}{2 \pi r_{1}} \frac{1}{\sin ^{2} \phi} .
\end{aligned}
$$

Substituting the principal radii, ie., $r_{1}, r_{2}$ into Eq.1 will give governing equations for the catnary shells of revolution.

Once we obtain the shear force $Q_{\phi}$ and rotation $\chi$, one can compute all other quantities, such as $N_{\phi \phi}, M_{\phi \phi}$, as well as $u, w$. Obviously, the governing equations are complicated and hard to be solved analytically.

\subsection{Displacement formulation of the shells of revolution}

Although Reissner-Meissner mixed formulations have some advantages, they cannot be used for vibration and nonlinear problems. Therefore, it is desirable to have a displacement formulations for centenary shells of revolution.

For convenience of readers, all quantities notation and definition in this section please refers to well-known book of J.N. Reddy 2007 (Reddy, 2007). For the shells of revolution shown in Fig. 4, the positions of points on the middle surface will be determined by the angles $\theta$ and $\varphi$. Further, let $r_{1}$ be the radius of curvature of the meridian and $r_{2}$ the radius of curvature of the normal section, tangential to the parallel circle. This second radius is equal to the segment of the perpendicular to the middle surface between this surface and the axis of the shells.

Regarding the forces shown in Fig.4, the balance equations are

$$
\begin{array}{r}
\frac{d}{d \phi}\left(r N_{\phi \phi}\right)-N_{\theta \theta} r_{1} \cos \phi+r Q_{\phi}+r_{1} r f_{\phi}=0, \\
\frac{d}{d \phi}\left(r Q_{\phi}\right)-r_{1} r\left(\frac{N_{\phi \phi}}{r_{1}}+\frac{N_{\theta \theta}}{r_{2}}\right)+r_{1} r f_{\zeta}=0,
\end{array}
$$

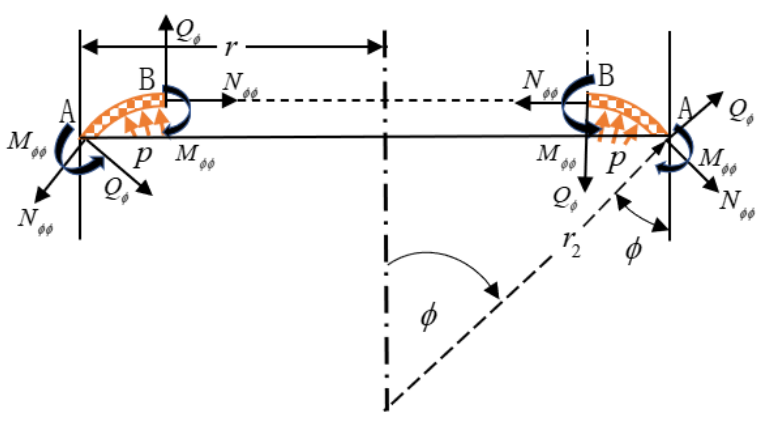

Figure 4: Geometry, loading, forces and moments.

where $r=r_{2} \sin \phi$, distributed loads $f_{\phi}, f_{\zeta}$ along $\phi, \zeta$ direction, and shear force

$$
Q_{\phi}=\frac{1}{r_{1} r} \frac{d}{d \phi}\left(r M_{\phi \phi}\right)-\frac{1}{r} \cos \phi M_{\theta \theta}
$$

where the resultant membranae forces are $N_{\phi \phi}=K\left(\varepsilon_{\phi \phi}+\right.$ $\left.v \varepsilon_{\theta \theta}\right), N_{\theta \theta}=K\left(v \varepsilon_{\phi \phi}+\varepsilon_{\theta \theta}\right)$, and resultant bending moments are $M_{\phi \phi}=B\left(\kappa_{\phi \phi}+v \kappa_{\theta \theta}\right), M_{\theta \theta}=B\left(\nu \kappa_{\phi \phi}+\kappa_{\theta \theta}\right)$; The membranae strains are $\varepsilon_{\phi \phi}=\frac{1}{r_{1}}\left(\frac{d u}{d \phi}+w\right), \varepsilon_{\theta \theta}=\frac{1}{r_{2}}(u \cot \phi+$ $w)$, and change of curvature are $\kappa_{\phi \phi}=\frac{1}{r_{1}} \frac{d \varphi_{\phi}}{d \phi}, \kappa_{\theta \theta}=\frac{\cot \phi}{r_{2}} \varphi_{\phi}$, where total rotation $\varphi_{\phi}=\frac{1}{r_{1}}\left(u-\frac{d w}{d \phi}\right)$; and membranae stiffness $K=\frac{E h}{1-v^{2}}$, bending stiffness $B=\frac{E h^{3}}{12\left(1-v^{2}\right)}$, thickness $h$, Young modulus $E$ and Poisson's ratio $v$.

With the above strains and curvature change, the resultant membranae force and bending moments can be expressed in terms of displacements $u, w$ as follows:

$$
\begin{aligned}
N_{\phi \phi} & =K\left[\frac{1}{r_{1}}\left(\frac{d u}{d \phi}+w\right)+v \frac{1}{r_{2}}(u \cot \phi+w)\right], \\
N_{\theta \theta} & =K\left[v \frac{1}{r_{2}}(u \cot \phi+w)+\frac{1}{r_{1}}\left(\frac{d u}{d \phi}+w\right)\right], \\
M_{\phi \phi} & =B\left[\frac{1}{r_{1}} \frac{d \varphi_{\phi}}{d \phi}+v \frac{\cot \phi}{r_{2}} \varphi_{\phi}\right] \\
& =B\left[\frac{1}{r_{1}} \frac{d}{d \phi}\left[\frac{1}{r_{1}}\left(u-\frac{d w}{d \phi}\right)\right]+v \frac{\cot \phi}{r_{2}} \frac{1}{r_{1}}\left(u-\frac{d w}{d \phi}\right)\right], \\
M_{\theta \theta} & =B\left[v \frac{1}{r_{1}} \frac{d \varphi_{\phi}}{d \phi}+\frac{\cot \phi}{r_{2}} \varphi_{\phi}\right] \\
& =B\left[v \frac{1}{r_{1}} \frac{d}{d \phi}\left[\frac{1}{r_{1}}\left(u-\frac{d w}{d \phi}\right)\right]+\frac{\cot \phi}{r_{2}} \frac{1}{r_{1}}\left(u-\frac{d w}{d \phi}\right)\right],
\end{aligned}
$$

Substituting the shear force $Q_{\phi}$ in Eq.6 into Eq.5 and pro- 
duce

$$
\begin{aligned}
& \frac{d}{d \phi}\left(r N_{\phi \phi}\right)-N_{\theta \theta} r_{1} \cos \phi \\
& +\frac{1}{r_{1}}\left[\frac{d}{d \phi}\left(r M_{\phi \phi}\right)-r \cos \phi M_{\theta \theta}\right]+r_{1} r f_{\phi}=0, \\
& \frac{d}{d \phi}\left[\frac{1}{r} \frac{d}{d \phi}\left(r M_{\phi \phi}\right)-\cos \phi M_{\theta \theta}\right] \\
& -r_{1} r\left(\frac{N_{\phi \phi}}{r_{1}}+\frac{N_{\theta \theta}}{r_{2}}\right)+r_{1} r f_{\zeta}=0,
\end{aligned}
$$

The Eq. 8 can be further simplified by substituting the constitutive relations into Eq.8, which will generate a final equations that is a six order ordinary differential equation system about displacement $u(\phi)$ and $w(\phi)$.

$$
\begin{aligned}
& K \frac{d}{d \phi}\left[r\left[\frac{1}{r_{1}}\left(\frac{d u}{d \phi}+w\right)+v \frac{1}{r_{2}}(u \cot \phi+w)\right]\right] \\
& -K r_{1} \cos \phi\left[v \frac{1}{r_{2}}(u \cot \phi+w)+\frac{1}{r_{1}}\left(\frac{d u}{d \phi}+w\right)\right] \\
& +\frac{B}{r_{1}} \frac{d}{d \phi}\left(r\left[\frac{1}{r_{1}} \frac{d}{d \phi}\left[\frac{1}{r_{1}}\left(u-\frac{d w}{d \phi}\right)\right]+v \frac{\cot \phi}{r_{2}} \frac{1}{r_{1}}\left(u-\frac{d w}{d \phi}\right)\right]\right) \\
& -\frac{B}{r_{1}} r \cos \phi\left[v \frac{1}{r_{1}} \frac{d}{d \phi}\left[\frac{1}{r_{1}}\left(u-\frac{d w}{d \phi}\right)\right]+\frac{\cot \phi}{r_{2}} \frac{1}{r_{1}}\left(u-\frac{d w}{d \phi}\right)\right] \\
& +r_{1} r f_{\phi}=0,
\end{aligned}
$$

$B \frac{d}{d \phi}\left[\frac{1}{r} \frac{d}{d \phi}\left(r\left[\frac{1}{r_{1}} \frac{d}{d \phi}\left[\frac{1}{r_{1}}\left(u-\frac{d w}{d \phi}\right)\right]+v \frac{\cot \phi}{r_{2}} \frac{1}{r_{1}}\left(u-\frac{d w}{d \phi}\right)\right]\right)\right.$ $\left.-\cos \phi\left[v \frac{1}{r_{1}} \frac{d}{d \phi}\left[\frac{1}{r_{1}}\left(u-\frac{d w}{d \phi}\right)\right]+\frac{\cot \phi}{r_{2}} \frac{1}{r_{1}}\left(u-\frac{d w}{d \phi}\right)\right]\right]$

$-r K\left[\frac{1}{r_{1}}\left(\frac{d u}{d \phi}+w\right)+v \frac{1}{r_{2}}(u \cot \phi+w)\right]$

$-K r \frac{r_{1}}{r_{2}}\left[\nu \frac{1}{r_{2}}(u \cot \phi+w)+\frac{1}{r_{1}}\left(\frac{d u}{d \phi}+w\right)\right]+r_{1} r f_{\zeta}=0$.

The above ODE system will be more complicated if taking into account of the principal radii. Obviously the ODE system has no analytical solution but numerical ones. To solve numerically, a general code is written in Maple and provided in the appendix.

\subsection{The sign convention of different formulations and physics units}

Although we can study the problem by either ReissnerMeissner formulation or displacement formulation, we should give an attention to the sign difference of respective quantities when comparing numerical results. For results validation purpose, we list the sign relations in Table 1.

For simplification of presentation of our results, physical units will not be plotted in all drawings (all physical units are listed in Table 2).
Table 1

Sign conventions and relations in different formulations

\begin{tabular}{c|c|c}
\hline Parameters & $\begin{array}{c}\text { Novozhilov (1959) } \\
\text { Reddy (2007) }\end{array}$ & Flügge (1973) \\
\hline Tangent displacement & $u$ & $u$ \\
\hline Normal displacement & $w$ & $w$ \\
\hline Total rotation & $\vartheta$ or $\varphi_{\phi}$ & $-\chi$ \\
\hline Bending moment & $M$ & $-M$ \\
\hline Resultant force & $T$ or $N$ & $N$ \\
\hline Shear force & $N$ or $Q$ & $-Q$ \\
\hline
\end{tabular}

Table 2

Physical units used in this paper

\begin{tabular}{c|c|c|c|c|c|c}
\hline$R$ & $a, b, c$ & $h$ & $E$ & $v$ & $M_{\phi}$ & $N_{\phi}$ \\
\hline$m$ & $m$ & $m$ & $N / m^{2}$ & 1 & $N$ & $N / m$ \\
\hline \hline$Q_{\phi}$ & $\sigma_{\phi}$ & $u$ & $w$ & & \\
\hline$N / m$ & $N / m^{2}$ & $m$ & $m$ & \\
Note: $N$ force physical unit and stands for Newton.
\end{tabular}

\section{The principal radii of curvature of the catenary shells of revolution}

The both of the above formulations of bending theory of shells are a general formulation for any shells of revolution. Clearly, we must derive the principal radii $r_{1}$ and $r_{2}$ if we wish to apply them to the catenary shells of revolution.

\subsection{The principal radii of curvature of the catenary shells of revolution 1}

The catenary shells of revolution 1 is formed by the revolution of a catenary $z=c \cosh \frac{x}{c}$ about the z-axis, where $c$ is a positive constant. The parametric equations for the catenary shells of revolution are then $x=c u \cos v, y=$ $c u \sin v, z=c \cosh u$.
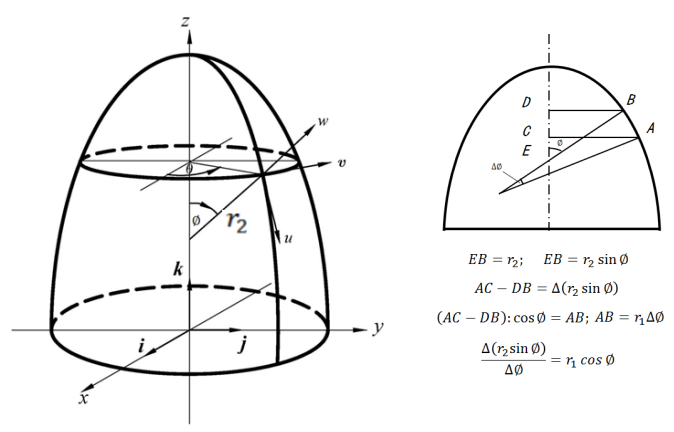

Figure 5: Geometry of the surface of revolution, the Lamé coefficients are $A_{1}=r_{1}, A_{2}=r_{2} \sin \phi$.

For the centenary surface of revolution shown in Fig. 5, the positions of points on the middle surface will be determined by the angles $\theta$ and $\phi$. Further, let $r_{1}$ be the radius of curvature of the meridian and $r_{2}$ the radius of curvature of the normal section, tangential to the parallel circle. This second radius is equal to the segment of the perpendicular to the middle surface between this surface and the axis of the torus.

If we denote $f=z-c \cosh \frac{x}{c}$, the principal radii can be 
obtained as follows:

$$
\begin{aligned}
& r_{1}=\left[1+\left(\frac{d z}{d x}\right)^{2}\right]^{3 / 2}\left(\frac{d^{2} z}{d x^{2}}\right)^{-1}=c \cosh ^{2} \frac{x}{c}, \\
& r_{2}=x\left(\frac{d z}{d x}\right)^{-1} \sqrt{1+\left(\frac{d z}{d x}\right)^{2}}=x \operatorname{coth} \frac{x}{c} .
\end{aligned}
$$

The above principal radii can be converted to another format that is expressed in the meridian angle $\phi$. From Fig.5, we have the tangent relation $\tan (\phi)=\frac{d z}{d x}$. With this relation, together with the equation $z=c \cosh \frac{x}{c}$, we can find $x(\phi)$ and $y(\phi)$ that are expressed in terms of meridian angle $\phi$. Hence we can find the 2 nd principal radius of the parallel circle as

$$
r_{2}=c \frac{\sinh ^{-1}(\tan \phi)}{\sin \phi} .
$$

Applying the relation of Gauss-Codazzi, $\frac{d r_{2} \sin \phi}{d \phi}=r_{1} \cos \phi$, we can obtain the meridian radius as follows

$$
r_{1}=\frac{c}{\cos ^{2} \phi} .
$$

The two principal curvatures are

$$
\begin{aligned}
& \kappa_{1}=\frac{1}{r_{1}}=\frac{\cos ^{2} \phi}{c}, \\
& \kappa_{2}=\frac{1}{r_{2}}=\frac{1}{c} \frac{\sin \phi}{\sinh ^{-1}(\tan \phi)} .
\end{aligned}
$$

and the Gauss curvature is

$$
K=\kappa_{1} \kappa_{2}=\frac{1}{c^{2}} \frac{\sin \phi \cos ^{2} \phi}{\sinh ^{-1}(\tan \phi)} .
$$

and the mean curvature is

$$
H=\frac{1}{2}\left(\kappa_{1}+\kappa_{2}\right)=\frac{1}{c}\left(\cos ^{2} \phi+\frac{\sin \phi}{\sinh ^{-1}(\tan \phi)}\right) .
$$

The curvatures are shown in Fig.7.

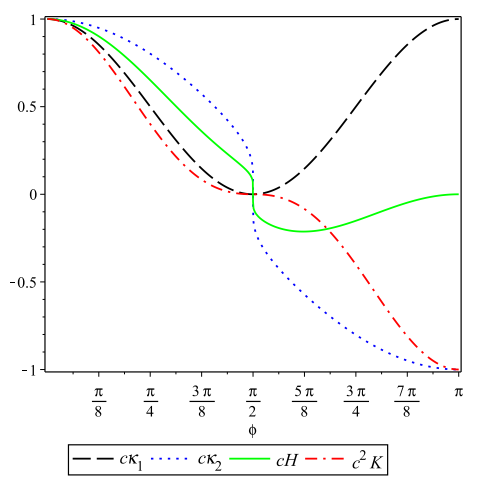

Figure 6: The curvatures of the catenary shells of revolution 1.

Since the mean curvature $H \neq 0$, therefore the catenary surface of revolution 1 is not a minimal surface.

\subsection{The principal radii of curvature of the catenary shells of revolution 2}

The catenary shells of revolution 2 is formed by the revolution of a catenary $x=c \cosh \frac{z}{c}$ about the z-axis, where $c$ is a positive constant. The parametric equations are then $x=c \cosh u \cos v, y=c \cosh u \sin v, z=c u$.

If we denote $f=x-c \cosh \frac{z}{c}$, the principal radii can be obtained as follows:

$$
\begin{aligned}
& r_{1}=\left[1+\left(\frac{d z}{d x}\right)^{2}\right]^{3 / 2}\left(\frac{d^{2} z}{d x^{2}}\right)^{-1}=-c \cosh ^{2} \frac{z}{c}, \\
& r_{2}=x\left(\frac{d z}{d x}\right)^{-1} \sqrt{1+\left(\frac{d z}{d x}\right)^{2}}=c \cosh ^{2} \frac{z}{c} .
\end{aligned}
$$

The above principal radii can be converted to another format that is expressed in the meridian angle $\phi$. From Fig.5, we have the tangent relation $\tan (\phi)=\frac{d z}{d x}$. With this relation, together with the equation $x=c \cosh \frac{z}{c}$, we can find $x(\phi)$ and $y(\phi)$ that are expressed in terms of meridian angle $\phi$.

$$
\begin{aligned}
& z=c \sinh ^{-1}\left(\frac{1}{\tan \phi}\right), \\
& x=\frac{c}{\sin \phi} .
\end{aligned}
$$

Hence we can find the 2nd principal radius of the parallel circle as

$$
r_{2}=\frac{x}{\sin \phi}=\frac{c}{\sin ^{2} \phi} .
$$

Applying the relation of Gauss-Codazzi, $\frac{d r_{2} \sin \phi}{d \phi}=r_{1} \cos \phi$, we can obtain the meridian radius as follows

$$
r_{1}=-\frac{c}{\sin ^{2} \phi} .
$$

The two principal curvatures are

$$
\begin{gathered}
\kappa_{1}=\frac{1}{r_{1}}=-\frac{\sin ^{2} \phi}{c}, \\
\kappa_{2}=\frac{1}{r_{2}}=\frac{\sin ^{2} \phi}{c} .
\end{gathered}
$$

and the Gauss curvature is

$$
K=\kappa_{1} \kappa_{2}=-\frac{\sin ^{4} \phi}{c^{2}} .
$$

and the mean curvature is

$$
H=\frac{1}{2}\left(\kappa_{1}+\kappa_{2}\right)=0 .
$$

The curvatures are shown in Fig.7.

Since the mean curvature $H=0$, therefore the catenary surface of revolution 2 is a minimal surface. 


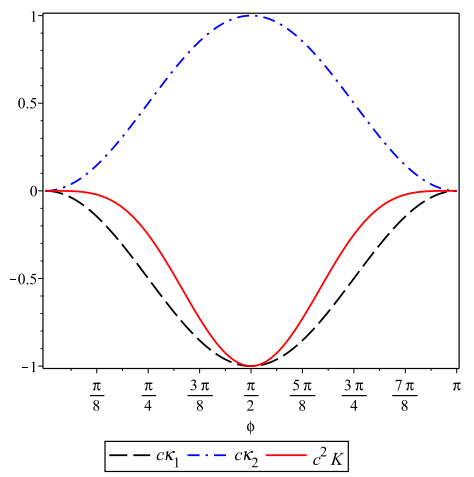

Figure 7: The curvatures of the catenary shells of revolution 2.

\section{Numerical studies for the deformation and stress of a truncated catenary shell of revolution 1}

For a truncated catenary shell of revolution at $x=b$ loaded with distributed unit bending moment $M=1[N]$ and free at $x=a$ the loading condition is shown in Fig. 8 .

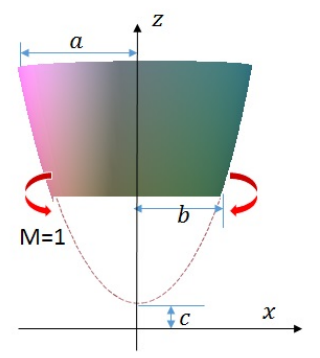

Figure 8: A truncated catenary shell of revolution under selfbalance distributed bending moment $M=1$

According to the tangent relation $\tan \phi=\sinh \frac{x}{c}$, hence

$$
\begin{aligned}
& \left.\phi\right|_{x=a}=\arctan \left(\sinh \frac{a}{c}\right) \\
& \left.\phi\right|_{x=b}=\arctan \left(\sinh \frac{b}{c}\right) .
\end{aligned}
$$

\subsection{Numerical results based on the displacement formulations}

For displacement formulations, the boundary conditions are:

$$
\begin{aligned}
& \phi=\left.\phi\right|_{x=a}: N_{\phi \phi}=0, Q_{\phi}=0, M_{\phi \phi}=0, \\
& \phi=\left.\phi\right|_{x=b}: N_{\phi \phi}=0, Q_{\phi}=0, M_{\phi \phi}=1[N] .
\end{aligned}
$$

Adapting the displacement formulations, some numerical results are shown in Fig. 9.

The results indicate that all quantities such as bending moments, surface forces, shear force, and displacement are little effected by the change of $b$.
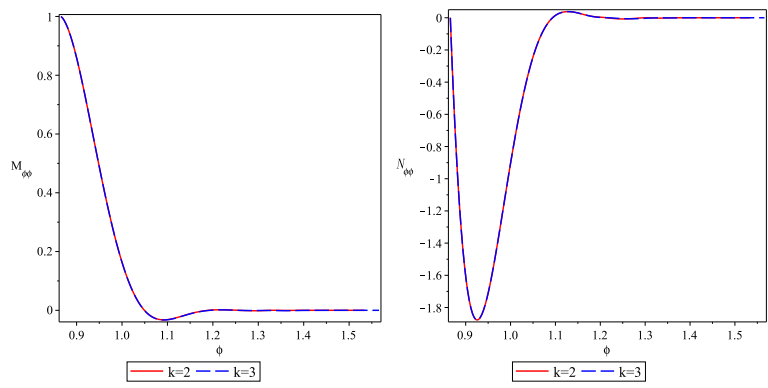

(a) Bending moment $M_{\phi \phi}$

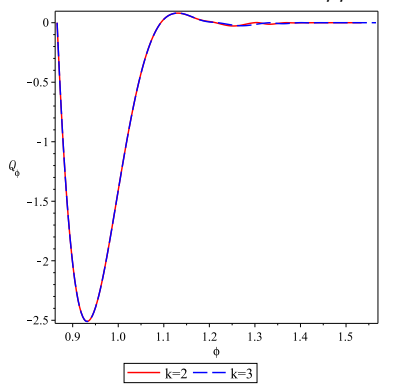

(c) Shear force $Q_{\phi}$ (b) Membranes force $N_{\phi \phi}$

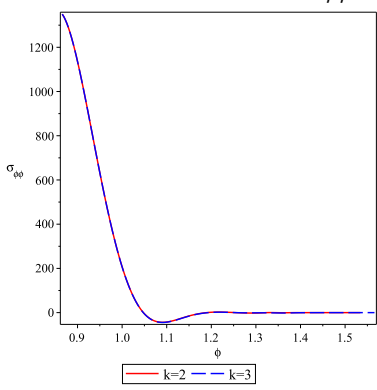

(d) Stress $\sigma_{\phi \phi}$
Figure 9: Displacement formulations for the catenary shells of revolution with data: $c=1[\mathrm{~m}], b=1[\mathrm{~m}], a=2 \mathrm{k}, \mathrm{h}=$ $c / 15[\mathrm{~m}], E=2.0 \times 10^{11} \mathrm{~N} / \mathrm{m}^{2}, v=0.3, M=1[\mathrm{Nm}]$ and $k=1,2,3$.

\subsection{Numerical results based on the Reissner-Meissner formulations}

According the the sign convention in Table 1, For the Reissner-Meissner formulations, the boundary conditions are:

$$
\begin{aligned}
& \phi=\left.\phi\right|_{x=a}: Q_{\phi}=0, M_{\phi \phi}=0, \\
& \phi=\left.\phi\right|_{x=b}: Q_{\phi}=0, M_{\phi \phi}=-1[N] .
\end{aligned}
$$

Some numerical results are shown in Fig. 13.

\subsection{Results validation}

According the the sign convention in Table 1, namely The difference of sign convention: $Q_{\phi}($ Displacement-formulations $)=$ $-Q_{\phi}$ (Reissner-Meissner-formulations), the result for the shear force comparison is presented in Fig.11.

The numerical simulations show that displacement formulation agrees with Reissner-Meissner's formulation very well, which provides a good supportive evidence about the correctness of our both formulation and simulation.

\section{Numerical studies for the deformation and stress of a catenary shell of revolution 2}

For a catenary shell of revolution at $y=b$ loaded with distributed unit bending moment $M=1[N]$ and free at $y=$ $b$ the loading condition is shown in Fig. 12.

According to the catenary equation: $y=c \cosh \frac{z}{c}$, we have $b=c \cosh \frac{z(b)}{c}$, leads to $z(b)=c \cosh ^{-1} \frac{b}{c}$. Since the tangent relation for this shell is $\tan \phi=\frac{1}{\sinh \frac{z}{c}}$, hence the 

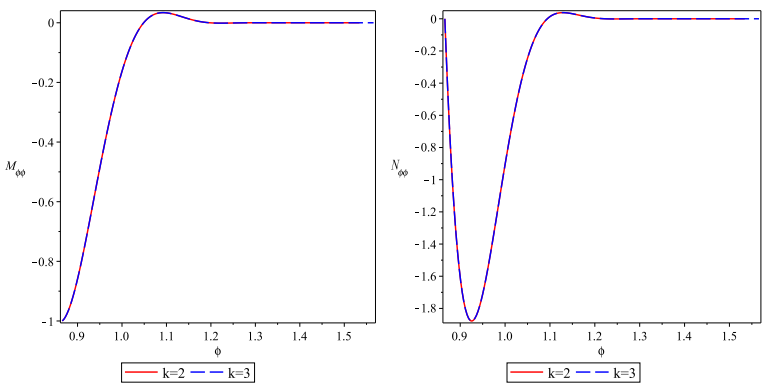

(a) Bending moment $M_{\phi \phi}$

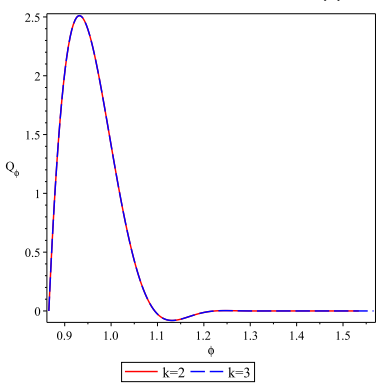

(c) Shear force $Q_{\phi}$ (b) Membranes force $N_{\phi \phi}$

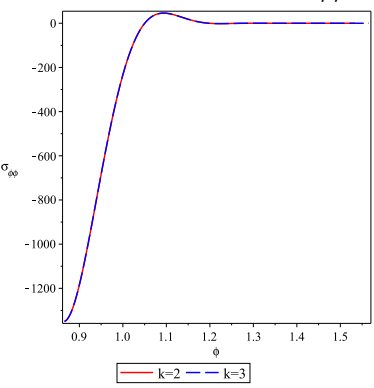

(d) Stress $\sigma_{\phi \phi}$

Figure 10: Reissner-Meissner formulations for the catenary shells of revolution with data: $c=1[\mathrm{~m}], b=1[\mathrm{~m}], a=2 \mathrm{k}, \mathrm{h}=$ $c / 15[\mathrm{~m}], E=2.0 \times 10^{11} \mathrm{~N} / \mathrm{m}^{2}, v=0.3, M=1[\mathrm{~N}]$ and $k=$ $1,2,3$.

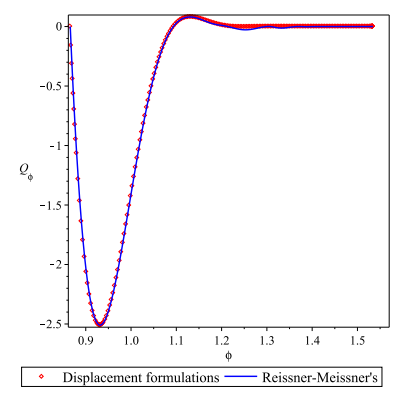

Figure 11: Shear force $Q_{\phi}$ comparison for the catenary shells of revolution with data: $c=1[\mathrm{~m}], b=1[\mathrm{~m}], a=4, h=$ $c / 15[\mathrm{~m}], E=2.0 \times 10^{11} \mathrm{~N} / \mathrm{m}^{2}, v=0.3, M=1[\mathrm{~N}]$. The difference of sign convention: $Q_{\phi}($ Displacement-formulation $)=$ $-Q_{\phi}($ Reissner-formulation $)$

corresponding angle are

$$
\begin{aligned}
& \phi_{a}=\arctan \left(\frac{1}{\sinh \frac{z(b)}{c}}\right) \\
& \phi_{b}=\pi-\phi_{a} .
\end{aligned}
$$

According the the sign convention in Table 1, for the Reissner-Meissner formulations, the boundary conditions are:

$$
\begin{aligned}
& \phi=\left.\phi\right|_{x=a}: Q_{\phi}=0, M_{\phi \phi}=-1[N], \\
& \phi=\left.\phi\right|_{x=b}: Q_{\phi}=0, M_{\phi \phi}=0 .
\end{aligned}
$$

Some numerical results are shown in Fig. 13.

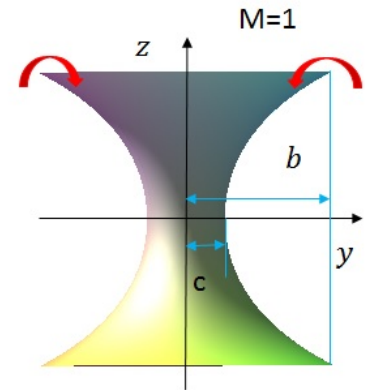

Figure 12: A catenary shell of revolution under self-balance distributed bending moment $M=1$
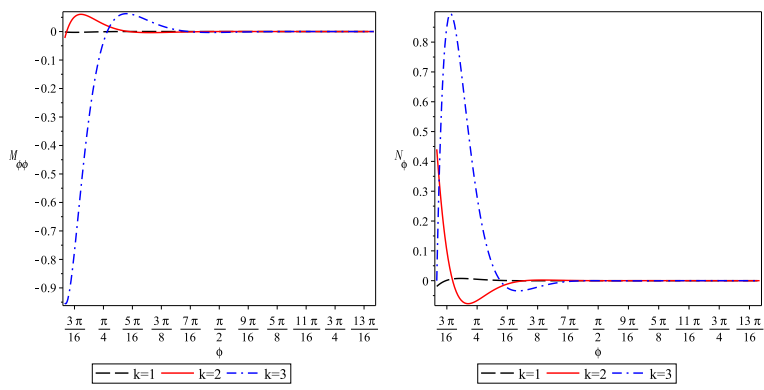

(a) Bending moment $M_{\phi \phi}$

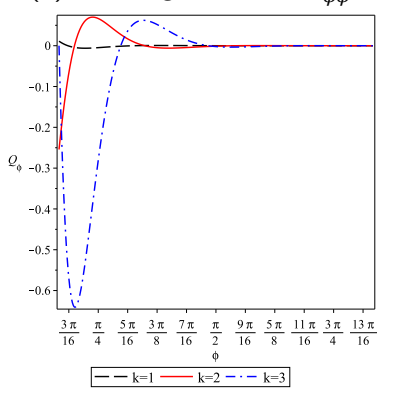

(b) Membranes force $N_{\phi \phi}$

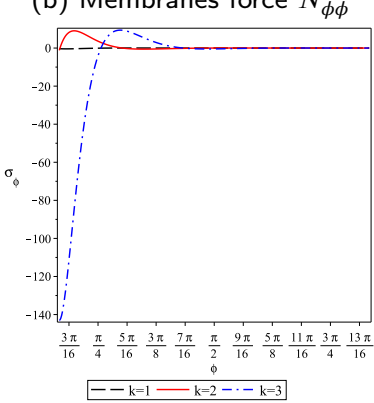

(c) Shear force $Q_{\phi}$

(d) Stress $\sigma_{\phi \phi}$

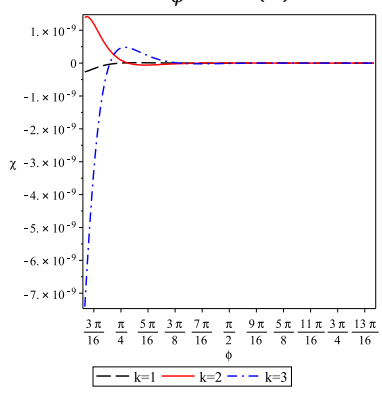

(e) Total meridian rotation $\chi$

Figure 13: Reissner-Meissner formulations for the catenary shells of revolution with data: $c=k[\mathrm{~m}], b=6[\mathrm{~m}], h=$ $c / 15[\mathrm{~m}], E=2.0 \times 10^{11} \mathrm{~N} / \mathrm{m}^{2}, v=0.3, M=1[\mathrm{~N}]$ and $k=1,2,3$. 


\section{The catenary shell of revolution vs. the spherical shells}

To compare the catenary shell of revolution and the spherical shells, let's us consider a semi-spherical shell in Fig.14 with radius $R$.

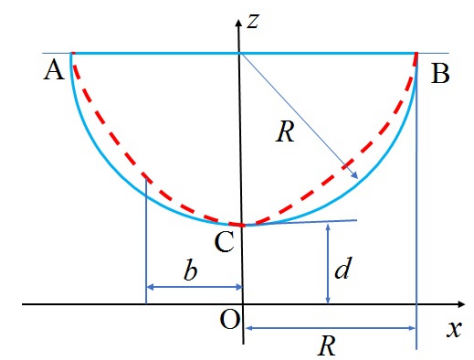

Figure 14: The spherical shell (blue) and the catenary shells of revolution (red): $d=0.618735305 R$.

To make the catenary shell of revolution to fit the spherical shell as much close as possible, we propose a design as shown in Fig.14, where both shells share a common top AB and bottom C. For this design, we have a geometrical relation

$$
d+R=d \cosh \frac{R}{d}
$$

Solving this implicit equation of $d$, we find

$$
d=0.618735305 R \text {. }
$$

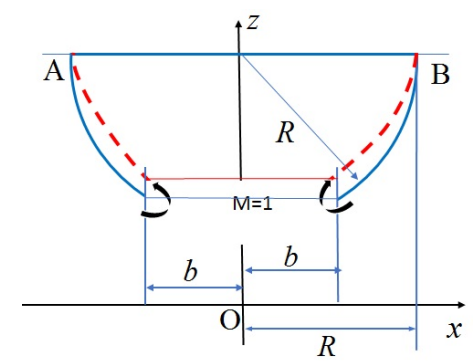

Figure 15: The loading and boundary conditions of the spherical shell (blue) and the catenary shells of revolution (red): $d=0.618735305 R$.

We will cut a hole along $x=b$ as shown in Fig. 15 . Both the spherical shell and the catenary shell of revolution are acted by a self-balance distributed unit bending moment $M=-1[N]$ around edge at $x=b$, while their bottom $\mathrm{AB}$ are free. Hence, the boundary conditions of the spherical are:

$$
\begin{aligned}
\phi=\left.\phi\right|_{x=R} & : Q_{\phi}=0, M_{\phi \phi}=0, \\
\phi=\left.\phi\right|_{x=b}: Q_{\phi} & =0, M_{\phi \phi}=-1[N],
\end{aligned}
$$

and the boundary conditions of the catenary shell of revolution are:

$$
\begin{gathered}
\phi=\left.\phi\right|_{x=R}=\arctan \left(\sinh \frac{R}{d}\right): \\
Q_{\phi}=0, M_{\phi \phi}=0, \\
\phi=\left.\phi\right|_{x=b}=\arctan \left(\sinh \frac{b}{d}\right): \\
Q_{\phi}=0, M_{\phi \phi}=-1[N] .
\end{gathered}
$$

The numerical results for both shells are carried out and presented in Fig.16.

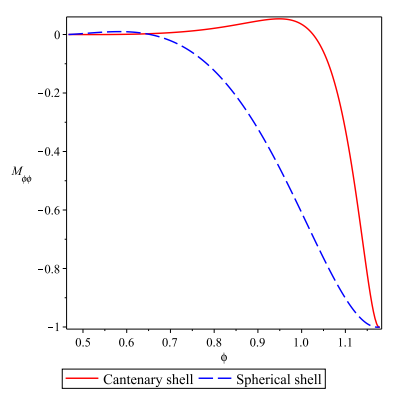

(a) Bending moment $M_{\phi \phi}$

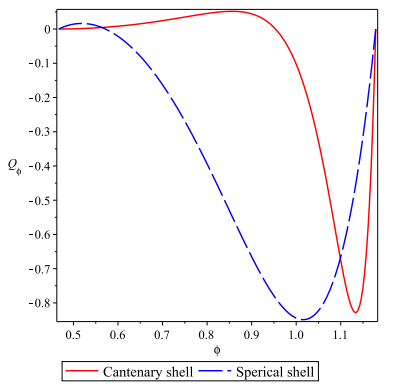

(b) Shear force $Q_{\phi}$

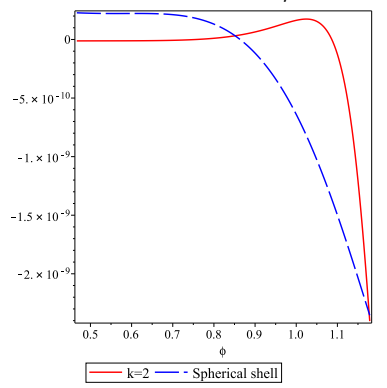

(c) Meridian rotation $\chi$

Figure 16: The catenary shells of revolution: $R=2[\mathrm{~m}], b=$ $0.3 R[\mathrm{~m}], d=0.618735305 R[\mathrm{~m}], h=R / 15[\mathrm{~m}], E=2.0 \times$ $10^{11} \mathrm{~N} / \mathrm{m}^{2}, v=0.3, M=1[N]$; The spherical shell: $R=$ $2[\mathrm{~m}], b=0.3 R[\mathrm{~m}], h=R / 15[\mathrm{~m}], E=2.0 \times 10^{11} \mathrm{~N} / \mathrm{m}^{2}, v=$ $0.3, M=1[N]$.

All results are shown in Fig.16 clearly indicate that the most region of the catenary shells of revolution have much less stress and deformation than the corresponding spherical shell, in other words, the most region of the catenary shells of revolution is almost in the membrane state except some boundary effect. We have tried different cases and all investigations point to an important conclusion that the mechanics of the catenary shells of revolution does much better than the spherical shells. 


\section{Analytical investigation on the catenary shells of revolution 2}

The Reissner-Meissner formulations of the catenary shells of revolution 1 are too complicate due to their odd principal radii. While the principal radii of the catenary shells of revolution 2 are relative simpler: $r_{1}=-\frac{c}{\sin ^{2} \phi}$ and $r_{2}=\frac{c}{\sin ^{2} \phi}$. With them we get $g(\phi)=0$, and the Reissner-Meissner equations are given as follows:

$$
\begin{aligned}
& L_{1}(\chi)+\frac{v}{c} \sin ^{2} \phi \chi=\frac{c}{B} \frac{Q_{\phi}}{\sin ^{2} \phi} \\
& c L_{1}\left(\frac{Q_{\phi}}{\sin ^{2} \phi}\right)-v Q_{\phi}+K\left(1-v^{2}\right) \chi=0,
\end{aligned}
$$

where the differential operator is

$$
L_{1}(:)=\frac{1}{c}\left[\sin ^{2} \phi \frac{d^{2}}{d \phi^{2}}(:)+\sin \phi \cos \phi \frac{d}{d \phi}(:)-\cos ^{2} \phi(:)\right] .
$$

If we set $\xi=\sin \phi$, the above equation system can be transferred to

$$
\begin{aligned}
& L(\chi)+v \xi^{2} \chi=\frac{c^{2}}{B} \frac{Q_{\phi}}{\xi^{2}}, \\
& L\left(\frac{Q_{\phi}}{\xi^{2}}\right)-v Q_{\phi}+K\left(1-v^{2}\right) \chi=0,
\end{aligned}
$$

where the operator

$$
L(:)=\xi^{2}\left(1-\xi^{2}\right) \frac{d^{2}}{d \xi^{2}}(:)+\xi \frac{d}{d \xi}(:)-\left(1-\xi^{2}\right)(:) .
$$

In the general case it is necessary to solve the equations Eq.47 as it stands by numerical integration.

\subsection{Solutions for the Poisson ratio $v=0$}

We make further progress toward simpler equations if we may assume the Poisson ratio $v=0$, an assumption often considered legitimate for reinforced concrete structures. The above equations can be reduced to a single equation

$$
L L(\chi)+c^{2} \frac{K}{B} \chi=0
$$

and

$$
L L\left(\frac{Q_{\phi}}{\xi^{2}}\right)+c^{2} \frac{K}{B} \frac{Q_{\phi}}{\xi^{2}}=0,
$$
tions

Those equations may be split into two second-order equa-

$$
L\left(\chi_{1}\right)+i c \sqrt{\frac{K}{B}} \chi_{1}=0 .
$$

and

$$
L\left(\chi_{2}\right)-i c \sqrt{\frac{K}{B}} \chi_{2}=0 .
$$

They can be further converted to the Heun's equation (Ronveaux, 1995), which is one of the hypergeometric equation, and from the general theory of this type of differential equations we may obtain the following solutions of Eq.51

$$
\chi=\chi_{1}+\chi_{2}
$$

where

$$
\begin{aligned}
& \chi_{1}=C_{1} \xi^{\alpha}\left(x^{2}-1\right)^{3 / 2} f_{1}+C_{2} \xi^{-\alpha}\left(x^{2}-1\right)^{3 / 2} f_{2} \\
& \chi_{2}=C_{3} \xi^{\beta}\left(x^{2}-1\right)^{3 / 2} f_{3}+C_{4} \xi^{-\beta}\left(x^{2}-1\right)^{3 / 2} f_{4},
\end{aligned}
$$

where $C_{k}(k=1,2,3,4)$ are integration constants and parameters

$$
\begin{aligned}
& \alpha=\left(1+i c \sqrt{\frac{K}{B}}\right)^{1 / 2}, \\
& \beta=\left(1-i c \sqrt{\frac{K}{B}}\right)^{1 / 2},
\end{aligned}
$$

and the functions $f_{k}(k=1,2,3,4)$ are expressed by the hypergeometric functions $F(\alpha, \beta, \gamma, x)$ (Marsden, Sirovich and Antman eds, 1991) as follows

$$
\begin{aligned}
& f_{1}=F\left(\alpha_{1}, \beta_{1}, \gamma_{1}, \xi^{2}\right) \\
& f_{2}=F\left(\alpha_{2}, \beta_{2}, \gamma_{2}, \xi^{2}\right) \\
& f_{3}=F\left(\alpha_{3}, \beta_{3}, \gamma_{3}, \xi^{2}\right) \\
& f_{4}=F\left(\alpha_{4}, \beta_{4}, \gamma_{4}, \xi^{2}\right),
\end{aligned}
$$

and the parameters are

$$
\begin{aligned}
& \alpha_{1}=\frac{1}{2} \alpha+\frac{\sqrt{5}+5}{4}, \beta_{1}=\frac{1}{2} \alpha-\frac{\sqrt{5}-5}{4}, \\
& \gamma_{1}=1+\alpha
\end{aligned}
$$

and

$$
\begin{aligned}
& \alpha_{2}=\frac{1}{2} \alpha+\frac{\sqrt{5}+5}{4}, \beta_{2}=-\frac{1}{2} \alpha-\frac{\sqrt{5}-5}{4}, \\
& \gamma_{2}=1-\alpha .
\end{aligned}
$$

and

$$
\begin{aligned}
& \alpha_{3}=\frac{1}{2} \beta-\frac{\sqrt{5}-5}{4}, \beta_{3} \quad=\frac{1}{2} \beta+\frac{\sqrt{5}+5}{4}, \\
& \gamma_{3}=1+\beta
\end{aligned}
$$

and

$$
\begin{aligned}
& \alpha_{4}=\frac{1}{2} \beta-\frac{\sqrt{5}-5}{4}, \beta_{4}=-\frac{1}{2} \beta+\frac{\sqrt{5}+5}{4}, \\
& \gamma_{4}=1-\beta .
\end{aligned}
$$




\subsection{Approximation in the vicinity of the apex}

In the vicinity of $\phi \rightarrow 0, \sin \phi$ and $\cos \phi$ may be expanded into a Laurent series. and if $\phi$ is small enough, we may approximate $\sin \phi$ and $\cos \phi$ by $\phi$ and 1, respectively.

When this is done in the coefficients of Eq.45, it reads

$$
\begin{aligned}
& L(\chi)+\nu \phi^{2} \chi=\frac{c^{2}}{B} \frac{Q_{\phi}}{\phi^{2}} \\
& L\left(\frac{Q_{\phi}}{\phi^{2}}\right)-v Q_{\phi}+K\left(1-v^{2}\right) \chi=0,
\end{aligned}
$$

where the differential operator is

$$
L=\phi^{2} \frac{d^{2}}{d \phi^{2}}+\phi \frac{d}{d \phi}-1 .
$$

In the general case it is necessary to solve the equations Eq.63 as it stands by numerical integration. Similarly, we can also make further progress toward simpler equations if we may assume the Poisson ratio $v=0$, whose exact solutions can be easily obtained as follows.

$$
\begin{aligned}
\chi & =\frac{i c}{\sqrt{B K}}\left(c_{1} \phi^{-\beta}+c_{2} \phi^{\beta}-c_{3} \phi^{-\alpha}-c_{4} \phi^{\alpha}\right), \\
Q_{\phi} & =\phi^{2}\left(c_{1} \phi^{-\beta}+c_{2} \phi^{\beta}+c_{3} \phi^{-\alpha}+c_{4} \phi^{\alpha}\right) .
\end{aligned}
$$

where $c_{k}(k=1,2,3,4)$ are integration constants and the parameters $\alpha, \beta$ are given in Eq.56.

Although the above analytical solutions have not been used for our numerical studies, the purpose of of presenting the analytical results is to provide them to readers for their own further investigation and applications.

\section{Conclusions}

We have formulated an elastic catenary shell of revolution in terms of displacement and mixed functions and successfully solved some typical problems. To verify our formulation, we provide two computational codes in Maple and carried out some numerical simulations. The validation of our numerical results was confirmed and supported by both the mixed and displacement formulations. Our investigations show that both deformation and stress response of the catenary shell of revolution is sensitive to the parameter $c$ or $d$. Our numerical studies also reveal that the mechanics of the catenary shells of revolution does much better than the spherical shells. For a future perspective, the nonlinear deformation, buckling and vivration behaviours of the catenary shells of revolution should be investigated.

\section{Acknowledgement:}

The author appreciates my students: Mr. Jie Wei, for preparation of Fig.4 and 14; Mr. Wen Dang for drawing of Fig.5.

\section{Data availability:}

The data that support the findings of this study are available from the corresponding author upon reasonable request.

\section{Appendix 1: Maple code of displacement formulation of the catenary shells of revolution}

for $\mathrm{k}$ from 1 to $3 \mathrm{do}$ :

restart; with(plots);

for $\mathrm{k}$ to 2 do $\mathrm{c}:=1 ; \mathrm{a}:=2 * \mathrm{k} ; \mathrm{b}:=1 ; \mathrm{h}:=(1 / 15)^{*} \mathrm{c}$;

phia $:=\arctan (\sinh (\mathrm{a} / \mathrm{c}))$;

phib $:=\arctan (\sinh (\mathrm{b} / \mathrm{c}))$ :

nu $:=.3 ; \mathrm{E}:=2 * 10 \mathrm{e}+11$

$\mathrm{K}:=\mathrm{E}^{*} \mathrm{~h} /(-\mathrm{nu} * \mathrm{nu}+1)$;

$\mathrm{B}:=\mathrm{E}^{*} \mathrm{~h} * \mathrm{~h} * \mathrm{~h} /\left(12 *\left(-n u^{*} \mathrm{nu}+1\right)\right)$;

$\mathrm{q} 1:=0 ; \mathrm{qn}:=0 ; \mathrm{F}:=1$;

$\mathrm{R} 1:=\mathrm{c} /(\cos (\mathrm{phi}) * \cos (\mathrm{phi}))$;

$\mathrm{R} 2:=\operatorname{arcsinh}(\tan (\mathrm{phi})) * \mathrm{c} / \sin (\mathrm{phi})$;

vartheta $:=0$;

$\mathrm{A} 1:=\mathrm{R} 1 ; \mathrm{A} 2:=\mathrm{R} 2 * \sin (\mathrm{phi})$;

$\mathrm{e} 11:=(\operatorname{diff}(\mathrm{u}(\mathrm{phi}), \mathrm{phi})) / \mathrm{A} 1+\mathrm{w}(\mathrm{phi}) / \mathrm{R} 1$;

$\mathrm{e} 12:=0 ; \mathrm{e} 13:=$-vartheta;

e22 := (diff(A2, phi) $) * u(p h i) /(A 1 * A 2)+w(p h i) / R 2 ;$

$\mathrm{e} 21:=0 ;$ e $23:=0$;

$\mathrm{x} 1:=\mathrm{e} 11+(1 / 2) * \mathrm{e} 13 * \mathrm{e} 13 ; \mathrm{x} 2:=\mathrm{e} 22$;

$\mathrm{k} 11:=(\operatorname{diff}($ vartheta, $\mathrm{phi})) / \mathrm{A} 1$;

$\mathrm{k} 12:=0 ; \mathrm{k} 13:=$-vartheta/R1;

$\mathrm{k} 22:=(\operatorname{diff}(\mathrm{A} 2, \mathrm{phi})) * \operatorname{vartheta} /(\mathrm{A} 1 * \mathrm{~A} 2)$;

$\mathrm{k} 21:=0 ; \mathrm{k} 23:=0$;

$\mathrm{y} 1:=\mathrm{e} 13 * \mathrm{k} 13+\mathrm{k} 11 ; \mathrm{y} 2:=\mathrm{k} 22$;

$\mathrm{T} 2:=\mathrm{K}^{*}\left(\mathrm{nu}^{*} \mathrm{x} 1+\mathrm{x} 2\right) ; \mathrm{M} 2:=\mathrm{B} *(\mathrm{nu} * \mathrm{y} 1+\mathrm{y} 2)$;

equ $1:=\mathrm{T} 1(\mathrm{phi})=\mathrm{K}^{*}\left(\mathrm{nu}^{*} \mathrm{x} 2+\mathrm{x} 1\right)$;

equ $2:=\mathrm{M} 1(\mathrm{phi})=\mathrm{B} *(\mathrm{nu} * \mathrm{y} 2+\mathrm{y} 1)$;

equ3 $:=\mathrm{N} 1($ phi $)=(\operatorname{diff}(\mathrm{A} 2 * \mathrm{M} 1(\mathrm{phi})$, phi $)$

-(diff(A2, phi) $) * \mathrm{M} 2) /(\mathrm{A} 1 * \mathrm{~A} 2)$;

equ4 $:=\operatorname{diff}(\mathrm{A} 2 * \mathrm{~T} 1$ (phi), phi)

$-(\operatorname{diff}(\mathrm{A} 2, \mathrm{phi})) * \mathrm{~T} 2+(\mathrm{N} 1(\mathrm{phi}) / \mathrm{R} 1+\mathrm{q} 1) * \mathrm{~A} 1 * \mathrm{~A} 2$

$-\mathrm{A} 1 * \mathrm{~A} 2 * \mathrm{~T} 1(\mathrm{phi}) *$ vartheta/R1 = 0 ;

equ $5:=\operatorname{diff}(\mathrm{A} 2 * \mathrm{~N} 1$ (phi), phi)

-(T1(phi)/R1+T2/R2)*A1*A2+qn*A $1 * \mathrm{~A} 2$

$+\operatorname{diff}(-\mathrm{A} 2 * \mathrm{~T} 1(\mathrm{phi}) * \mathrm{vartheta}, \mathrm{phi})=0$;

equs := equ1, equ2, equ3, equ4, equ5;

bc: $=\mathrm{T} 1($ phib $)=0, \mathrm{~N} 1($ phib $)=0, \mathrm{M} 1(\mathrm{phib})=1$,

$\mathrm{T} 1(\mathrm{phia})=0, \mathrm{~N} 1(\mathrm{phia})=0, \mathrm{M} 1(\mathrm{phia})=0$ :

sys:=equs,bc:

vars: $=\mathrm{u}(\mathrm{phi}), \mathrm{w}(\mathrm{phi}), \mathrm{T} 1(\mathrm{phi}), \mathrm{M} 1(\mathrm{phi}), \mathrm{N} 1(\mathrm{phi})$ :

nov: $=$ dsolve (sys, vars, numeric, abserr $=0.01$,output $=$ listprocedure $)$;

$\mathrm{M} 11[\mathrm{k}]:=\operatorname{rhs}(\operatorname{nov}[2])$

$\mathrm{Q} 1[\mathrm{k}]:=\operatorname{rhs}(\operatorname{nov}[3]):$

$\mathrm{N} 11[\mathrm{k}]:=\operatorname{rhs}(\operatorname{nov}[4])$ :

print(k);

od:

\section{Appendix 2: Maple code of Reissner-Meissner formulation of the catenary shells of revolution}

restart; with(plots);

for $\mathrm{k}$ from 1 to 3 do:

$\mathrm{R}:=2 * \mathrm{k} ; \mathrm{c}:=.618735305^{*} \mathrm{R}$;

$\mathrm{h}:=(1 / 15)^{*} \mathrm{R} ; \mathrm{b}:=.3 * \mathrm{R}$;

phib $:=\arctan (\sinh (\mathrm{R} / \mathrm{c}))$;

phic $:=\arctan (\sinh (\mathrm{b} / \mathrm{c}))$;

$\mathrm{nu}:=0.3 ; \mathrm{E}:=0.200 \mathrm{e} 12$;

$\mathrm{K}:=\mathrm{E}^{*} \mathrm{~h} /(-\mathrm{nu} * \mathrm{nu}+1)$;

$\mathrm{B}:=\mathrm{E} * \mathrm{~h} * \mathrm{~h} * \mathrm{~h} /\left(12 *\left(-n{ }^{*}\right.\right.$ nu+1) $)$;

$\mathrm{P}:=0$;

$\mathrm{r} 1:=\mathrm{c} /(\cos (\mathrm{phi}) * \cos (\mathrm{phi}))$;

$\mathrm{r} 2:=\operatorname{arcsinh}(\tan (\mathrm{phi}))^{*} \mathrm{c} / \sin (\mathrm{phi})$ :

odes $:=r 2 *(\operatorname{diff}(\operatorname{varkappa}(\mathrm{phi})$, phi, phi) $) / \mathrm{r} 1$

$+(\mathrm{r} 2 * \cot (\mathrm{phi}) / \mathrm{r} 1+\operatorname{diff}(\mathrm{r} 2 / \mathrm{r} 1$, phi $)$

$+\mathrm{r} 2 *(\operatorname{diff}(\mathrm{B}, \mathrm{phi})) /(\mathrm{r} 1 * \mathrm{~B})) *(\operatorname{diff}(\operatorname{varkappa}(\mathrm{phi})$, phi $))$

$-(\mathrm{r} 1 * \cot (\mathrm{phi}) / \mathrm{r} 2 * \cot (\mathrm{phi})$

+nu-nu*(diff(B, phi) $) * \cot ($ phi $) / \mathrm{B}) * \operatorname{varkappa}(\mathrm{phi})$

$=\mathrm{r} 1 * \mathrm{r} 2 * \mathrm{Q}(\mathrm{phi}) / \mathrm{B}, \mathrm{r} 2 *(\operatorname{diff}(\mathrm{r} 2 * \mathrm{Q}(\mathrm{phi}), \mathrm{phi}, \mathrm{phi})) / \mathrm{r} 1$

$+(\mathrm{r} 2 * \cot (\mathrm{phi}) / \mathrm{r} 1+\operatorname{diff}(\mathrm{r} 2 / \mathrm{r} 1, \mathrm{phi})$ 


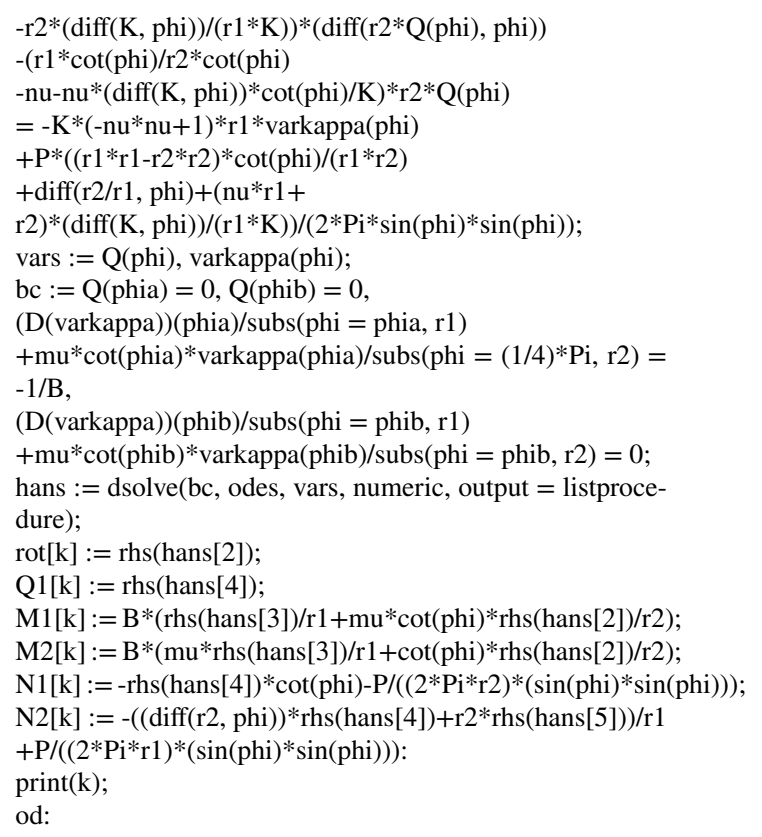

\section{References}

Audoly, B., Pomeau, Y., 2010. Elasticity and geometry. Oxford University Press.

Calladine, C.R., 1983. Theory of Shell Structures. Cambridge University Press.

Flügge, W., 1973. Stresses in shells. Springer Berlin Heidelberg. .

Gol'denveizer, A.L., 1961. Theory of Elastic thin Shells. Pergamon Press, New York.

Kraus, H., 1967. The Elastic Shells. John Wileys \& Sons..

Marsden, J.E., Sirovich, L., Antman eds, S.S., 1991. Hypergeometric Functions and Their Applications, Texts in Applied Mathematics 56. Springer-Verlag New York.

Meissner, E., 1915. über und elastizitat festigkeit dünner schalen. Vierteljahrschrift der Naturforschungs, Gesellschaft, Zurich. 60, 23-47.

Novozhilov, V.V., 1959. The theory of thin shells. P. Noordhoff.

Reddy, J.N., 2007. Theory and Analysis of Elastic Plates and Shells. CRC Press.

Reissner, H., 1912. Spannungen in kugel-schalen (kuppeln). Festschrift Heinrich Müller-Breslau., 181-193.

Ronveaux, A., 1995. Heun's Differential Equations. Oxford University Press.

Sun, B., 2012. Toroidal Shells. Nova Novinka, New York.

Sun, B., 2021. On symmetrical deformation of toroidal shell with circular cross-section. Thin-walled Structures 163, 107680.

Timoshenko, S., Woinowsky-Krieger, S., 1959. Theory of Plates and Shells. Mcgraw-Hill College, New York.

Ugural, A., 2018. Plates and shells : theory and analysis. McGraw-Hill Book Company.

Zingoni, A., 2017. Shell Structures in Civil and Mechanical Engineering. ICE Publishing. 